\title{
Le transport des protéines dans le noyau : les signaux de localisation nucléaire et leurs récepteurs
}

Les protéines nucléaires, synthétisées dans le cytoplasme comme toutes les protéines, sont adressées au noyau grâce à un signal de localisation nucléaire (NLS, nuclear localisation signal) constitué d'une courte séquence d'acides aminés basiques, parfois bipartite. Le NLS est reconnu par des protéines spécifiques - dont les nucléoporines - et, peut-être par leur intermédiaire, se fixe aux pores de la membrane nucléaire. En présence d'ATP, la protéine est alors transloquée dans le noyau, peutêtre grâce aux propriétés contractiles des nucléoporines.

\section{Valérie Schreiber Gilbert de Murcia Josiane Ménissier- de Murcia}

\section{ADRESSE}

V. Schreiber : allocataire de recherche Mrt. G. de Murcia : directeur de recherche au Cnrs. J. Ménissier-de Murcia : chargée de recherche au Cnrs. Institut de biologie moléculaire et ccllulaire du Cnrs associé à l'université Louis-Pastcur, laboratoire de biochimic II, 15, rue René-Descartes, 67084 Strasbourg a diversité des réactions biochimiques dans le noyau nécessite le passage d'un grand nombre de macromolécules à travers la membrane nucléaire. Elle sépare les fonctions de réplication et de transcription de l'ADN, de la fonction de traduction des ARN en protéines. L'avantage sélectif dû à la présence de la membrane nucléaire permet, en contrôlant l'importation de protéines, d'obtenir de rapides et fines modifications de concentration de celles-ci. Par exemple, certains facteurs protéiques qui existent en faible quantité dans la cellule, comme les facteurs de régulation de la réplication et de la transcription, doivent être impérativement présents dans le noyau en fonction de la progression du cycle cellulaire ou en réponse à un signal extérieur. Dans cette revue, nous résumons les connaissances actuelles concernant les signaux de localisation nucléaire et leurs récepteurs. Nous renvoyons les lecteurs désireux de s'informer sur les conséquences du contrôle de la translocation nucléaire des protéines au niveau de la régulation de la transcription génique, à l'article de synthèse de M. Piechaczyk et P. Roux, paru dans médecinel sciences [1].

L'enveloppe nucléaire est constituée d'une membrane externe, en continuité avec les membranes du réticulum endoplasmique, et d'une membrane interne, associée aux lamines, protéines présentes dans la matrice nucléaire servant de points d'ancrage à la chromatine [2]. Ces deux membranes sont fusionnées au niveau des pores nucléaires, structures supramoléculaires constituées de plus d'une centaine de protéines différentes. L'observation des pores au microscope électronique couplé au traitement d'images a permis d'obtenir des clichés de haute résolution : suivant un axe perpendiculaire à la surface de l'enveloppe nucléaire, le pore nucléaire présente une symétrie rotationnelle d'ordre 8 . Il se compose de deux anneaux (l'un du côté du cytoplasme, l'autre dirigé vers l'intérieur 
du noyau) reliés à un corps central par un ensemble de spicules. En réponse à un signal, ces anneaux se déformeraient et s'ouvriraient à la manière d'un double iris de diaphragme [3, 4]. Le transport de macromolécules à travers le pore nucléaire est bidirectionnel : entrée spécifique de protéines et sortie de sous-unités préribosomiques, de ribonucléoprotéines et d'ARNt (pour une revue voir [5]) (figure 1). Ces pores fonctionnent comme des tamis moléculaires : ils permettent le passage par diffusion passive de petites molécules comme les solvants et les ions. Théoriquement, les protéines de masse inférieure à 40-60 kDa peuvent diffuser passivement à l'intérieur du noyau. Cependant de petites protéines, comme les histones dont la synthèse et la présence dans le noyau sont requises en phase $\mathrm{S}$ du cycle cellulaire, bénéficient d'un mécanisme de transport facilité, ce qui accroît considérablement l'efficacité de leur passage dans le noyau. Ce même transport actif est mis en jeu lors du passage dans le noyau de protéines de masse supérieure. Ce mécanisme est décomposé en deux étapes : (1) la reconnaissance spécifique par un récepteur d'un signal de localisation nucléaire (NLS* : nuclear localization signal) présent sur la protéine à importer ; (2) la translocation à travers la membrane nucléaire; cette étape requiert une température physiologique et consomme de l'ATP [6].

Il faut noter que la présence d'un NLS sur la protéine caryophile est nécessaire, mais pas suffisante, pour assurer son passage dans le noyau : en effet, le signal peut être enfoui dans la macromolécule ou masqué par sa fixation à une protéine (protéine d'ancrage, voir figure 1). Une modification conformationnelle de la protéine à importer (phosphorylation, liaison à un effecteur...) ou de la protéine d'ancrage rend le NLS fonctionnel [7].

\footnotetext{
* Abréviations. NLS: signal de localisation nucléaire; $N B P$ : récepleurs des proléines à $N L S$; $R P N$ : ribonucléoprotéines; Ala $=A ; A r g=R$; $A s n=N ; A s p=D ; C y s=C ; G l n=Q$; $G l u=E ; G l y=G ;$ His $=H ;$ Ile $=I ;$ Leu $=L ;$ Lys $=K ;$ Mel $=M ; P h e=F$; Pro $=P ; \operatorname{Ser}=S ; \operatorname{Thr}=T ; \operatorname{Trp}=W ;$ Tyr $=Y:$ Val $=V$. $\mathrm{m} / \mathrm{s} n^{\circ} 2$, vol. 8, féviier 92
}

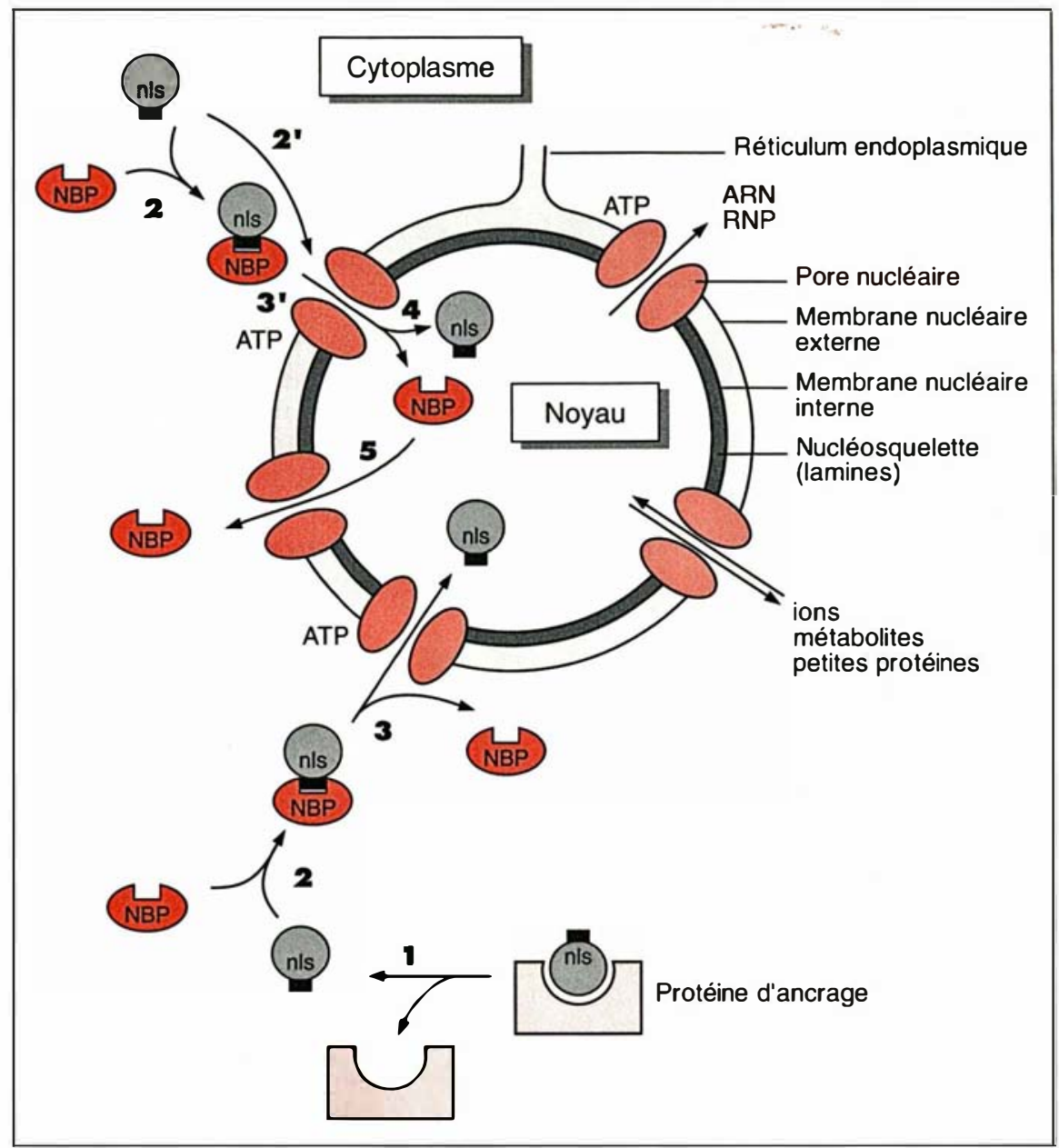

Figure 1. Schéma illustrant les différentes étapes qui régissent les échanges entre le cytoplasme et le noyau. Après synthèse dans le cytoplasme, la protéine caryophile est transportée dans le noyau. Plusieurs étapes peuvent régir son transport : la protéine peut être retenue dans le cytoplasme par une protéine d'ancrage (1); après dissociation du complexe sous l'effet de facteurs cytoplasmiques, la protéine se lie à un récepteur-transporteur (NBP, NLS-binding protein) (2) ou directement au' pore nucléaire (2'). Le récepteur se dissocie de la protéine sur le pore nucléaire qui la transporte dans le noyau (3), ou c'est le complexe protéine-récepteur qui est entièrement transporté dans le noyau ( $\left.3^{\prime}\right)$ où il se dissocie (4). Le récepteur peut être recyclé dans le cytoplasme (récepteur navette) (5) ou dégradé.

De nombreuses expériences ont suggéré l'existence des NLS. Nous donnerons ci-dessous quelques exemples qui illustrent ce fait.

- En 1975, Bonner [8] micro-injecte des protéines d'oocytes de Xenopus laevis dans des oocytes intacts : les protéines d'origine nucléaire sont transportées dans le noyau, tandis que les protéines d'origine cytoplasmique restent localisées dans le cytoplasme.

- En 1982, Dingwall montre que la nucléoplasmine, une protéine d'ori- gine nucléaire, micro-injectée dans le cytoplasme des oocytes de Xenopus laevis s'accumule dans le noyau [9]. En revanche, la nucléoplasmine protéolysée, tronquée de son extrémité Cterminale, est exclue du noyau, alors que ce fragment C-terminal microinjecté dans le cytoplasme est transporté rapidement dans le noyau. Ces résultats ont suggéré l'existence d'un signal de localisation nucléaire dans l'extrémité C-terminale de la nucléoplasmine. 


\section{RÉFÉRENCES}

1. Picchaczyk M, Roux P. Le contrôle de la translocation nucléairc des protéines : un niveau supplémentaire de régulation de l'activité des gènes. médecine/sciences $1990 ; 6$ : 803-6.

2. Nelson WG, Pienta KJ, Barrack ER, Coffey DS. The role of the nuclear matrix in the organization and function of DNA. Ann Rev Biophys Chem 1986; 15 : 457-75.

3. Stcwart M, Whytock S, Mills A. Association of gold-labelled nuclcoplasmin with the centres of ring components of Xenopus oocytes nuclear pore complexes. $J \mathrm{Mol}$ Biol $1990 ; 213$ : 575-82.

4. Akcy CW. Visualization of transportrelated configurations of the nuclear porc transporter. Biophys J 1990 ; 58 : 341-55.

5. Goldfarb D, Michaud N. Pathways for the nuclcar transport of proteins and RNAs. Trends Cell Biol 1991; 1 : 20-4.

6. Newmeycr DD, Forbes DJ. Nuclear import can be scparated into distinct steps in vitro: nuclcar porc binding and translocation. Cell 1988; 52 : 641-53.

7. Hunt T. Cytoplasmic anchoring proteins and the control of nuclear localization. Cell 1989 ; 59 : 949-51.

8. Bonner WM. Protein migration into nuclei. II. Frog oocyte nuclei accumulate a class of microin jected oocyte nuclear proteins and cxclude a class of microinjected cytoplasmic protcins. J Cell Biol 1975; 64 : 431-7.

9. Dingwall C, Sharnick SV, Laskcy RA A polypeptide domain that specifics migration of nuclcoplasmin into the nucleus. Cell 1982 ; 30 : 449-58.

10. Hall MN, Hereford L, Herskowitz I. Targeting of $E$. coli $\beta$-galactosidase to the nuclcus in ycast. Cell 1984; 36 : 1057-65. 11. Kalderon D, Richardson WD, Mark ham AF, Smith AE. Sequence requirements for nuclear location of simian virus 40 large T antigen. Nature 1984; 311 : 499-509.

12. Lanford RE, Butcl JS. Construction and characterization of an SV40 mutant defective in nuclear transport of $\mathrm{T}$ antigen. Cell $1984 ; 37$ : 801-13.

13. Silver P, Goodson H. Nuclear protein transport. Crit Rev Biochem Mol Biol 1989 24 : 419-35.

14. Robcrts B. Nuclear location signalmediated protein transport. Biochim Biophys Acta 1989 ; 1008 : 263-80.

15. Garcia-Bustos J, Heitman J, Hall MN. Nuclear protcin localization. Biochim Biophys Acta 1991; 1071 : 83-101.

16. Robcrts BL, Richardson WD, Smith AE. The effect of protein context on nuclcar location signal function. Cell 1987 50 : 465-75.

17. Richardson WD, Robcrts BL, Smith AE. Nuclear location signals in polyoma virus large-T. Cell 1986; 44 : 77-85.

18. Lanford RE, Kanda P, Kennedy $P$ Induction of nuclear transport with a synthe tic peptide homologous to the SV40 antigen
- Les 13 résidus $\mathrm{N}$-terminaux de la protéine de levure Mat $\alpha 2$ suffisent, lorsqu'ils sont fusionnés à la $\beta$ galactosidase, à cibler la protéine chimérique vers le noyau [10].

- Kalderon et ses collaborateurs [11] ont démontré que la séquence (126) PKKKRKV (132) dans l'antigène T du virus SV40, fusionnée à la pyruvate kinase permet le ciblage de la protéine recombinante dans le noyau. De plus, la mutation de la lysine en position 128 abolit totalement la caryophilicité de la protéine $[11,12]$.

\section{Les signaux} de localisation nucléaire

Pour caractériser un NLS, deux approches complémentaires ont été développées. La première est une approche "soustractive", dans laquelle le NLS hypothétique est soit délété, soit muté ponctuellement afin de montrer qu'il est nécessaire à la localisation nucléaire de la protéine. La seconde approche est dite " additive " : elle consiste à coupler le NLS putatif à une protéine indicatrice cytoplasmique afin de montrer qu'il est suffisant pour l'adressage de la protéine dans le noyau. Ce couplage peut être effectué soit par greffage chimique d'un peptide synthétique correspondant au NLS putatif sur la protéine indicatrice, soit par fusion de la séquence oligonucléotidique correspondante, avec le gène codant pour la protéine indicatrice. Ces constructions sont alors introduites dans les cellules soit par micro-injection, soit par transfection de l'ADN recombinant. La localisation de la protéine recombinante micro-injectée ou synthétisée in vivo pcut être détectée par immunofluorescence, par une réaction histochimique, par microscopie électronique ou encore par fractionnement subcellulaire. Un exemple d'approche additive est illustré figure 2, p. 138.

Depuis 1984, de nombreux NLS ont été étudiés (pour une revue voir [13, $14,15])$. Leurs séquences peptidiques sont rassemblées dans le Tableau I. Aucune séquence consensus n'émerge de la comparaison de ces différents NLS. Cependant, en règle générale, les NLS sont constitués de séquences basiques (riches en lysine et arginine) de 5 à 8 résidus, fréquemment bor- dées de glycine ou de proline (acides aminés permettant la formation d'un coude dans la séquence peptidique, ce qui pourrait contribuer à la bonne orientation du NLS vers l'extérieur de la protéine). Ils peuvent être localisés à tout endroit de la protéine, toutefois ils se situent toujours dans des zones hydrophiles [16] et, le cas échéant, dans des régions charnières séparant les domaines des protéines multifonctionnelles. On admet que ces signaux sont situés à la surface de la protéine nucléaire permettant ainsi leur reconnaissance par un récepteur. De plus, la présence d'un site hydrolysé par la trypsine au cœur de la séquence du NLS de la poly(ADPribose)polymérase (lysine 223) confirme la localisation de ce NLS à la surface de la protéine (Schreiber et al., en préparation). Enfin, les NLS, à la différence des peptides $\mathrm{NH}_{2}$-terminaux existant dans les séquences des protéines à excréter, ne sont pas clivés suite à la localisation nucléaire de la protéine.

Bien que la fonction de ciblage et de translocation des protéines puisse être assurée par un seul signal, ressemblant au NLS de l'antigène $T$ de SV40 (Tableau IA), certaines protéines nucléaires contiennent plusieurs NLS (Tableau IB). Par exemple, l'antigène $T$ du virus du polyome possède deux NLS qui coopèrent pour permettre l'entrée de la protéine dans le noyau [17] ; la caryophilicité de la protéine n'est abolie qu'après mutation simultanée des deux signaux. Dans ce cas, la présence de deux signaux fonctionnels permet à la protéine virale de rentrer plus rapidement dans le noyau. Par ailleurs, il avait déjà été montré par greffage chimique de NLS sur une protéine indicatrice, que la vitesse d'accumulation dans le noyau est proportionnelle au nombre de peptides greffés [18]. Enfin, la réitération d'un signal, même partiellement défectueux dans le transport, peut être suffisante pour promouvoir l'accumulation de la protéine dans le noyau [16]. Ces résultats indiquent que la multiplication de NLS faiblement actifs sur une protéine peut avoir un effet positif dans le routage des protéines dans le noyau.

D'autres protéines possèdent plusieurs NLS non équivalents. Ainsi, d'après 
Tableau I

LES SIGNAUX DE LOCALISATION NUCLÉAIRE

A. Protéines possédar
SV40 antigène-T
Adénovirus E1A
h. lamine A
SV40 VP1
h. PDGF chaîne A
Adénovirus pTP
d. sry $\delta$
I. histone 2B

A. Protéines possédant un NLS unique

B. Protéines possédant 2 NLS

I. protéine ribosomique L29

I. MAT $\alpha 2$

h. C-MYC

virus du polyome antigène-T
(123)

(279)

(412)

(1)

(192) DTGRPRESGKKRKRKRLKPT-(COOH)

(317) FVDRLPVRRRRRRVPPPP

(178) KSSDGEDRPTKKRVKQECTT

(24)
Références

[15]

[15]

[15]

[15]

[15]

[15]

[34]

[15]

\title{
C. Protéines possédant un NLS bipartite
}

\author{
$x$. nucléoplasmine \\ x. N1 \\ h. poly(ADP-ribose) polymérase \\ m. KIN 17 \\ *m. p53 \\ *h. p53 \\ ${ }^{*} \mathrm{~m}$. c-ABL \\ * $x$. NO38 \\ * la. récepteur des progestérones \\ ${ }^{*}$ r. récepteur des glucocorticoïdes
}

(14) KGRIGKHRKHPGG
$(140)$ VRILESWFAKNI
$(361)$ VLERQRRNELKRSF
(277) ATPPKKAREDPAP

Abréviations : $m$ : murin ; $r$ : rat ; 1 : levure ; $h$ : humain; la : lapin; $d$ : drosophile; $x$ : xénope.

Les motifs basiques sont représentés en gras. Les séquences soulignées suffisent à promouvoir l'accumulation dans le noyau d'une protéine traceur. II n'a pas été montré que les protéines marquées d'un (") possèdent un NLS bipartite, mais leur séquence primaire le suggère.

$[x]$ : Schreiber et al., en préparation.

Les chiffres entre parenthèses indiquent la position dans la protéine de la séquence donnée.

les travaux récents de Hall et al. [19], la protéine Mat $\alpha 2$, déjà citée, contiendrait deux NLS : une séquence N-terminale, initialement décrite, permettant la fixation à la membrane nucléaire et une séquence localisée entre les résidus 140 et 151 , fonctionnelle en l'absence de la première, responsable de la translocation de la protéine dans le noyau. Un autre exemple est illustré par les récepteurs des hormones glucocorticoïdes qui possèdent un NLS inductible et un autre constitutif. Le signal inductible est localisé dans le domaine de fixation de l'hormone [20]. En absence de glucocorticoïdes, le récepteur est cytoplasmique ; la fixation de l'hormone impose une modification conformationnelle telle que le NLS dépendant de l'hormone démasqué devient fonctionnel et que le complexe récepteur-glucocorticoïde migre dans le noyau. Le NLS constitutif, localisé entre les domaines de fixation à l'ADN (domaine $\mathrm{C}$ ) et de liaison à l'hormone (domaine E), est fonctionnel dans un récepteur dont le domaine $\mathrm{E}$ a été délété. Cette protéine mutée est toujours nucléaire, même en absence d'hormone.

Enfin, une nouvelle classe de NLS a été récemment définie : les NLS bipartites [21] (Tableau IC). Il s'agit de signaux constitués de deux motifs basiques interdépendants. Ces motifs de deux à six résidus sont séparés par une dizaine d'acides aminés. De courtes insertions ou délétions dans la région séparant ces motifs basiques pcuvent être tolérées. Mais aucune des deux régions prises séparément n'est capable d'assurer le transport de la protéine dans le noyau. Dans la nucléoplasmine de Xenopus laevis, la séquence du NLS est : (155) KR$\mathrm{X}_{10}-\mathrm{KKKK}$ (170). Une mutation ponctuelle dans chacun des motifs basiques diminue, mais n'abolit pas complètement, la localisation nucléaire de la protéine : en revanche, la mutation simultanée des deux motifs rend la protéine cytoplasmique. Le NLS de la protéine N1 de Xenopus laevis est également bipartite; sa séquence est : (532) RKKRK-X ${ }_{12}$-KKSK (552). La mutation de la lysine 534 ou 549 abolit la fonction du signal de localisation nucléaire [22]. Autre exemple, celui de la poly(ADP-ribose)polymérase humaine, enzyme associée aux mécanismes de réparation de l'ADN, dans laquelle les deux séquences basiques (206) KRK - X 11 -KKKSKK (226) sont également requises pour le ciblage de la protéine dans le noyau et incapables de fonctionner séparément. Dans cette enzyme, les résidus basiques 207, 208 et 222 sont importants pour le fonctionnement du NLS (Schreiber et al., en préparation). La protéine KIN17, protéine murine 


\section{RÉFÉRENCES}

19. Hall MN, Craik C, Hiraoka Y Homeodomain of yeast repressor $\alpha 2$ contains a nuclear localization signal. Proc Natl Acad Sci USA 1990 ; 87 : 6954-8.

20. Picard D, Yamamoto KR. Two signals mediate hormone-dependent nuclear localization of the glucocorticoid receptor. $E M B O$ J $1987 ; 6: 3333-40$

21. Robbins J, Dilworth SM, Laskey RA, Dingwall C. Two interdependent basic domains in nucleoplasmin nuclear targeting sequence : identification of a class of bipartite nuclear targeting sequence. Cell 1991; $64: 615-23$.

22. Kleinschmidt JA, Seiter A. Identification of domains involved in nuclear uptake and histone binding of protein N1 of Xenopus laevis oocytes. J Biol Chem 1988; 7 : 1605-14.

23. Angulo JF, Rouer E, Mazin A, et al. Identification and expression of the cDNA of KIN17, a zinc-finger gene located on mouse chromosome 2, encoding a new DNA-binding protein. Nucleic Acids Res $1991 ; 19: 5117-23$

24. Shaulsky G, Goldfinger N, BenZe'ev A, Rotter V. Nuclear accumulation of p53 protein is mediated by several nuclear localization signals and plays a role in tumorigenesis. Mol Cell Biol 1990; 10 6565-77.

25. Diller L, Kassel J, Nelson CE, et al. p53 functions as a cell cycle control protein in osteosarcomas. Mol Cell Biol 1990 ; 10 : $5772-81$

26. Rihs HP, Jans DA, Fan H, Peters R. The rate of nuclear cytoplasmic transport is determined by the casein kinase II site flanking the nuclear localization sequence of the SV40 T-antigen. EMBOJ $1991 ; 10$ : 633-9. 27. Adam SA, Lobl TJ, Mitchell M Gerace L. Identification of specific binding proteins for a nuclear location sequence. Nature 1989 ; 337 : 276-9.

28. Adam SA, Gerace L. Cytosolic proteins that specifically bind nuclear location signals are receptors for nuclear import. Cell 1991 $66: 837-47$

29. Yoneda $Y$, Imamoto-Sonobe $N$, Matsuoka $Y$, Iwamoto $R$, Kiho $Y$, Uchida $T$. Antibodies to Asp-Asp-Glu-Asp can inhibit transport of nuclear proteins into the nucleus. Science $1988 ; 242$ : 275-8.

30. Finlay DR, Forbes DJ. Reconstitution of biochemically altered nuclear pores : transport can be eliminated and restored. Cell $1990 ; 60: 17-27$

31. Dingwall C. Transport across the nuclear envelope : enigmas and explanations. BioEssays 1991; 13 : 213-8.

32. Nehrbass U, Kern H, Mutvei A, Horstmann H, Marshallsay B, Hurt EC. NSP1: a yeast nuclear envelope protein localized at the nuclear pores exerts its essential function by its carboxy-terminal domain. Cell 1990 ; 61 : 979-89.

33. Starr CM, Hanover JA. A common structural motif in nuclear pore proteins (nucleoporins). BioEssays 1991; 13 : 145-6. 34. Noselli S, Vincent A. A Drosophila nuclear localization signal included in a 18 amino acid fragment from the serendipity $\delta$ zinc finger protein. FEBS Lett 1991 . $280: 167-70$.

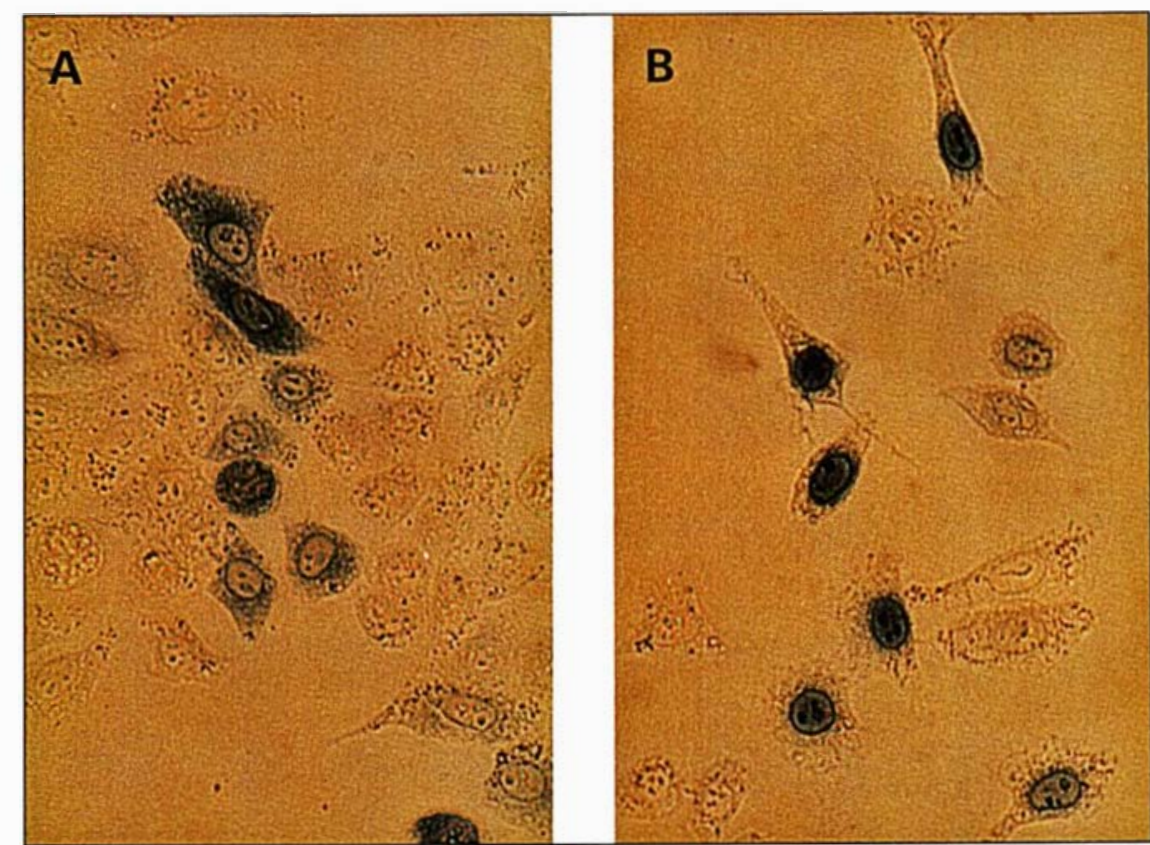

Figure 2. Mise en évidence d'un signal de localisation nucléaire par approche additive. La séquence nucléotidique codant pour le NLS de l'antigène $T$ de SV4O [(125)PPKKKRKVEDP(135)] a été clonée en phase avec le gène codant pour la $\beta$-galactosidase, dans le vecteur d'expression eucaryotique pCH 110 (Pharmacia). Des cellules Hela ont été transfectées, soit par le vecteur $p C H 110$, soit par le vecteur recombinant appelé pCH SV NLS. La localisation de la $\beta$-galactosidase est révélée par son activité enzymatique, en utilisant le X-gal comme substrat, ce qui engendre une coloration bleue. La $\beta$ galactosidase est localisée dans le cytoplasme des cellules transfectées par le vecteur $\mathrm{pCH} 110$ (figure 2A). La protéine chimérique a été ciblée dans le noyau des cellules transfectées par pCH SV NLS (figure 2B).

sérologiquement reliée à la protéine bactérienne RecA, possède également un NLS calqué sur ce modèle [23]. $A$ posteriori, il apparaît maintenant que de nombreux signaux de localisation nucléaire, précédemment décrits comme étant constitués d'un seul motif basique rappelant le NLS de l'antigène $T$ de SV40, possèdent en fait un NLS bipartite. Ainsi, le suppresseur de tumeur p53 de souris possède un signal en position 313-318 indispensable à la localisation nucléaire de la protéine [24]. Dans cette séquence, la lysine 316 est essentielle à cette fonction. Dans la protéine humaine de séquence très proche, une double mutation rend la protéine mutée cytoplasmique [25] ; l'une des mutations porte sur une lysine située en position - 12 par rapport au motif basique principal, l'autre concerne une glycine localisée à 39 résidus en amont de la première mutation. Ce dernier changement n'ayant probablement pas d'effet sur le phénotype de la protéine mutée, les protéines p53 seraient donc potentiellement des protéines à NLS bipartite.

Des études cinétiques ont été effectuées pour le NLS de l'antigène $T$ de SV40 [26]. L'accumulation dans le noyau, d'une protéine chimérique comportant ce NLS (résidus 126-134) passe de 15 heures à 8-10 minutes lorsque le NLS est flanqué de sa région $\mathrm{N}$-terminale, c'est-à-dire des résidus 111 à 125 . Cette région comporte quatre sites de phosphorylation. C'est la phosphorylation par la caséine kinase II des sérines 111 et 112 qui entraîne une augmentation de la vitesse de transport. On peut constater dans le Tableau I que les NLS possèdent généralement des résidus 
susceptibles d'être phosphorylés à proximité de leurs séquences basiques.

\section{Les récepteurs des protéines nucléaires}

La caractérisation biochimique et fonctionnelle des récepteurs des NLS (NBP* : NLS-binding proteins) est une des clés majeures du mécanisme qui régit le transport des protéines dans le noyau. Le modèle le plus simple prévoyait que les NBP étaient associés aux pores nucléaires, mais de nombreux travaux récents ont montré que les récepteurs sont des protéines navettes que l'on peut détecter dans le cytosol et dans des extraits nucléaires $[27,28]$. Le transport s'effectuerait en plusieurs étapes ; la première consisterait en la fixation de la protéine à importer sur un récepteur cytoplasmique (voir figure 1). Le complexe récepteur-protéine serait alors reconnu par un système assurant le transport, localisé au centre du pore nucléaire et responsable de la translocation du complexe dans le noyau. Le complexe serait alors dissocié et le récepteur libre pourrait être recyclé dans le cytoplasme ou dégradé.

Initialement, les NBP potentiels ont été détectés par l'utilisation des méthodes d'affinité :

- marquage par des peptides de synthèse portant des séquences NLS documentées [27] :

- immunodétection par un anticorps dirigé contre la séquence DDDED [29] supposée portée par le récepteur reconnaissant le NLS de l'antigène T de SV40 (KKKRK).

De cette façon, plusieurs NBP ont été décrits et caractérisés. Certains devraient jouer un rôle dans la translocation des protéines; en particulier, la famille des nucléoporines, glycoprotéines portant des groupements $\mathrm{N}$ acétylglucosamine. En effet, le transport dans le noyau des protéines nucléaires peut être inhibé par des anticorps monoclonaux dirigés contre les nucléoporines ainsi que par l'agglutinine de germe de blé, une lectine qui se fixe sur la partie glucosidique de la glycoprotéine [6]. De plus, dans un système de reconstitution in vitro de noyaux, des pores nucléaires traités à l'agglutinine de germe de blé, et par conséquent dépourvus de nucléoporines fonction- nelles, ne sont plus capables d'assurer le transport de protéines [30]. Ces expériences illustrent bien le rôle déterminant des nucléoporines dans ce processus. Récemment, les ADNc codant pour trois nucléoporines ont été clonés chez le rat (nucléoporine p62) et chez la levure (NUP1 et NSP1) (pour une revue voir [31]). Ces protéines sont organisées en différents domaines dont l'un est probablement structuré en hélice $\alpha$. Des études de mutants de NSP1 ont montré que cette région en hélice $\alpha$ est essentielle pour assurer la translocation des protéines dans le noyau [32]. Ce domaine porte des motifs répétés particuliers, caractéristiques des protéines filamenteuses; en effet, des motifs similaires de sept résidus sont présents dans des régions en hélice $\alpha$ de la myosine, de la tropomyosine et de la kératine. Les protéines assurant le transfert des protéines dans le noyau pourraient donc également adopter une structure filamenteuse. Cette observation est en faveur de l'idée que les protéines nucléaires seraient transloquées dans le noyau par un mécanisme mettant en jeu la contraction consommant de l'énergie (comme dans le cas de la myosine) de ces protéines filamenteuses [33].

\section{Conclusion}

Les signaux de localisation nucléaire portés par la protéine à importer dans le noyau ont été découverts et leurs séquences abondamment décrites. Mais il reste, aujourd'hui, difficile de comprendre, sur la simple base des données de la séquence peptidique, comment un (ou des) récepteur(s) protéique(s) reconnaissent une si grande variété de NLS. L'étude structurale fine de ces séquences devrait nous renseigner sur leur conformation. Plusieurs hypothèses sont à l'étude : existe-t-il un motif structural partagé par tous ces signaux malgré les diversités de séquences? Dans le cas contraire, les récepteurs peuvent-ils s'adapter à différentes structures de NLS ou se sont-ils " spécialisés " pour reconnaître certaines classes de NLS ? Cette dernière phase dans l'étude du ciblage de protéines dans le noyau devrait permettre d'élucider le mécanisme moléculaire qui régit ce transport

\section{Summary}

Nuclear protein transport : nuclear localization signals and their receptors

Nuclear pore complexes provide the route by which nuclear proteins travel from the cytoplasm to the nucleus of a cell. Proteins are translocated to nucleus because they contain one or more short sequences of basic amino acids which act as nuclear location signal (NLS). In this paper we describe the partition of the NLScontaining proteins into three different classes - (i) the SV large $\mathrm{T}$-like proteins, (ii) proteins having two or more independent targeting signals and (iii) those containing a bipartite NLS. Proteins to be imported into the nucleus associate with the nuclear pore complexes, possibly via NLSbinding proteins (NBP). NBPs comprise a class of glycoproteins : the nucleoporins and, to date, the cDNAs of three such proteins have been cloned and each shown to have a common structural motif containing heptad repeat unit. In addition we address the possible function and organization of the NBP-like proteins in the nuclear pore complexes of the cell.

\section{Remerciements}

Les auteurs tiennent à remercier le professeur G. Dirheimer pour son encouragement constant. Ce travail a été subventionné par l'Association pour la recherche contre le cancer et par la Fédération nationale des ligues contre le cancer et la Ligue nationale contre le cancer (comité départemental du Haut-Rhin)

\section{TIRÉS A PART}

J. Ménissier-de Murcia. 